PROCEEDINGS OF THE

AMERICAN MATHEMATICAL SOCIETY

Volume 35, No. 1, September 1972

\title{
CONTRACTIBLE HILBERT CUBE MANIFOLDS
}

\section{T. A CHAPMAN ${ }^{1}$}

\begin{abstract}
In this note we give an example of a contractible Hilbert cube manifold which cannot be embedded as an open subset of the Hilbert cube $Q$ so that its complement (in $Q$ ) lies in a face of the boundary of $Q$. This example provides a negative answer to a question raised by the author.
\end{abstract}

1. Introduction. Let the Hilbert cube $Q$ be represented by $\prod_{i=1}^{\infty} I_{i}$, where each $I_{i}$ is the closed interval $[0,1]$, and let $W=\left\{\left(x_{i}\right) \in Q \mid x_{1}=1\right\}$. By a Hilbert cube manifold (or $Q$-manifold) we mean a separable metric space for which each point has a closed neighborhood homeomorphic to $Q$. In this paper we will only be concerned with noncompact contractible $Q$-manifolds, since all compact contractible $Q$-manifolds are homeomorphic to $Q$ [3]. If $A \subset W$ is a closed set, then it is clear that $Q \backslash A$ is a contractible $Q$-manifold. In general define a $Q$-manifold to be of type $Q$ provided that it is homeomorphic to $Q \backslash A$, for some closed $A \subset W$. We remark that any closed set $A \subset W$ has Property $\mathrm{Z}$ in $Q$ (in the sense of Anderson [1]), but this concept will not be used in this paper. In fact we use no infinite-dimensional topology other than some elementary topological properties of $Q$.

The $Q$-manifolds of type $Q$ form a particularly distinguished collection because of the following representation and characterization theorems.

THEOREM 1 ([3]). If $X$ is a $Q$-manifold of type $Q$, then there exists a countable locally-finite simplicial complex $K$ such that $X$ is homeomorphic to $|K| \times Q$.

THEOREM 2 ([5]). If $X$ and $Y$ are $Q$-manifolds of type $Q$ which have the same proper homotopy type, then $X$ and $Y$ are homeomorphic.

(See $\S 2$ for definitions concerning the proper category.)

Attempting to extend these results to the class of all contractible $Q$ manifolds one is naturally led to the following question (see Question 4 of [4] and $Q M 5$ of [1]):

Question. Is every contractible $Q$-manifold of type $Q$ ?

Received by the editors December 1, 1971.

AMS 1969 subject classifications. Primary 5540; Secondary 5450.

Key words and phrases. Hilbert cube, Hilbert cube manifold, Whitehead's example.

${ }^{1}$ Supported in part by NSF grant GP-28374. 
It is obvious that an affirmative answer to this question would extend Theorem 1 and Theorem 2 to the class of all contractible $Q$-manifolds.

The objective of this note is to answer the above question negatively by giving an example of a contractible $Q$-manifold which is not of type $Q$. If $M$ is Whitehead's well-known example of an open contractible 3manifold which is not homeomorphic to $E^{3}$ [7], then we prove that $M \times Q$ is a contractible $Q$-manifold which is not of type $Q$. To be more precise (and in the language of $\S 2$ ) we prove that $M \times Q$ is not proper homotopically dominated by any $Q$-manifold of the form $Q \backslash A$, where $A \subset W$ is closed. We also remark that this example sharpens the following result of [3] concerning decompositions of contractible $Q$-manifolds into open sets of type $Q$ : Any contractible $Q$-manifold can be written as the union of tw'o dense open sets, each one of which is of type $Q$.

The question of extending Theorem 1 and Theorem 2 to the class of all contractible $Q$-manifolds remains open. The example given in this paper merely rules out one method of attack.

2. Proper maps. We give here some basic definitions concerning proper maps, and in Lemma 2.1 we establish a result which will be used in $\S 3$.

For spaces $X$ and $Y$ we say that a map (i.e. a continuous function) $f: X \rightarrow Y$ is proper provided that $f^{-1}(C)$ is compact, for all compact sets $C \subset Y$. Two proper maps $f, g: X \rightarrow Y$ are said to be proper homotopic (written $f \simeq_{n} g$ ) provided that there exists a proper map $F: X \times[0,1] \rightarrow Y$ which satisfies $F(x, 0)=f(x)$ and $F(x, 1)=g(x)$, for all $x \in X$. (We reserve the symbol $\simeq$ for the usual notion of homotopic maps.) We say that spaces $X$ and $Y$ have the same proper homotopy type provided that there exist proper maps $f: X \rightarrow Y$ and $g: Y \rightarrow X$ such that $g \circ f \simeq_{p}$ id $_{X}$ and $f \circ g \sim_{p}$ id $_{Y}$ (where $\mathrm{id}_{Y}$ and $\mathrm{id}_{Y}$ denote the identity maps of $X$ and $Y$, respectively). As an analogue of the concept of homotopy domination we say that a space $X$ proper homotopically dominates a space $Y$ provided that there exist proper maps $f: X \rightarrow Y$ and $g: Y \rightarrow X$ such that $f \circ g \sim{ }_{p} \mathrm{id}_{Y}$.

The following result will be needed in $\$ 3$. It pinpoints a property of locally compact metric spaces which are proper homotopically dominated by $Q \backslash A$, for some closed $A \subset W$.

Lemma 2.1. Let $X$ be a locally compact metric space which is proper homotopically dominated by $Q \backslash A$, for some closed $A=W$. Then for each compact set $K_{1} \subset X$ there exists a compact set $K_{2} \subset X$ containing $K_{1}$ and there exists a map

$$
F:\left(X \backslash K_{2}\right) \times[0,1] \rightarrow X: K_{1}
$$

such that $F(x, 0)=x$ and $F(x, 1) \in K_{2} \backslash K_{1}$, for all $x+x ; K_{2}$. 
Proof. We will first establish the above property for the space $Q \backslash A$. Thus let $K_{1}^{\prime} \subset Q \backslash A$ be a compact set ; we want to find a compact set $K_{2}^{\prime} \subset$ $Q \backslash A$ which contains $K_{1}^{\prime}$ and a map $F^{\prime}:\left(Q \backslash\left(A \cup K_{2}^{\prime}\right)\right) \times[0,1] \rightarrow Q \backslash\left(A \cup K_{1}^{\prime}\right)$ such that $F^{\prime}(x, 0)=x$ and $F^{\prime}(x, 1) \in K_{2}^{\prime} \backslash K_{1}^{\prime}$, for all $x \in Q \backslash\left(A \cup K_{2}^{\prime}\right)$.

Note that $Q \backslash K_{1}^{\prime}$ is an open set containing $A$. Choose $V$ to be a relatively open subset of $W$ such that $A \subset V \subset \mathrm{Cl}_{W}(V) \subset Q \backslash K_{1}^{\prime}$, where $\mathrm{Cl}_{W}(V)$ is the closure of $V$ in $W$. Let $\mathrm{Bd}_{W}(V)$ be the topological boundary of $V$ in $W$ and let $p: W \rightarrow \prod_{i=2}^{x, 2} I_{i}$ be projection. Clearly there exists a map $r: \mathrm{Cl}_{W}(V) \rightarrow$ $[0,1]$ such that $r(V) \subset(0,1), r\left(\operatorname{Bd}_{W}(V)\right)=\{1\}$, and $[r(x), 1] \times\{p(x)\} \subset$ $Q \backslash K_{1}^{\prime}$, for all $x \in \mathrm{Cl}_{W}$. $(V)$. Let

$$
U=\bigcup\{(r(x), 1] \times\{p(x)\} \mid x \in V\},
$$

which is an open subset of $Q$ containing $A$. Then we put $K_{2}^{\prime}=Q \backslash U$.

Note that $U \mid A=Q \backslash\left(A \cup K_{2}^{\prime}\right)$ and define $F^{\prime}:(U \backslash A) \times[0,1] \rightarrow Q \backslash\left(A \cup K_{1}^{\prime}\right)$ by setting $F^{\prime}((s, p(x)), t)=((1-t) s+\operatorname{tr}(x), p(x)) \in[r(x), 1] \times\{p(x)\}$, for all $x \in V,(s, p(x)) \in((r(x), 1] \times\{p(x)\}) \backslash A$, and $t \in[0,1]$. It is clear that $F^{\prime}$ fulfills our requirements.

Passing to the general case let $f: X \rightarrow Q \backslash A$ and $g: Q \backslash A \rightarrow X$ be proper maps such that $g \circ f \simeq_{p}$ id $_{X}$ and let $K_{1} \subset X$ be a given compact set. Since $g$ is proper there exists a compact set $K_{1}^{\prime} \subset Q \backslash A$ such that $g\left(Q \backslash\left(A \cup K_{1}^{\prime}\right)\right) \cap K_{1}=$ $\varnothing$. Using the special case treated above there exist a compact set $K_{2}^{\prime} \subset$ $Q \backslash A$ containing $K_{1}^{\prime}$ and a map $F^{\prime}:\left(Q \backslash\left(A \cup K_{2}^{\prime}\right)\right) \times[0,1] \rightarrow Q \backslash\left(A \cup K_{1}^{\prime}\right)$ such that $F^{\prime}(x, 0)=x$ and $F^{\prime}(x, 1) \in K_{2}^{\prime} \backslash K_{1}^{\prime}$, for all $x \in Q \backslash\left(A \cup K_{2}^{\prime}\right)$.

Since $f$ is proper and $g \circ f \simeq_{p}$ id $_{X}$ we can find a compact set $K_{2}^{*} \subset X$ containing $K_{1}$ such that $f\left(X \backslash K_{2}^{*}\right) \subset Q \backslash\left(A \cup K_{2}^{\prime}\right)$ and we can find a map $G:\left(X \backslash K_{2}^{*}\right) \times[0,1] \rightarrow X \backslash K_{1}$ such that $G(x, 0)=x$ and $G(x, 1)=g \circ f(x)$, for all $x \in X \backslash K_{2}^{*}$. Define $F^{*}:\left(X \backslash K_{2}^{*}\right) \times[0,1] \rightarrow X \backslash K_{1}$ by setting

$$
\begin{aligned}
F^{*}(x, t) & =G(x, 2 t) & & 0 \leqq t \leqq \frac{1}{2}, \\
& =g \cdot F^{\prime}(f(x), 2 t-1) & & \frac{1}{2} \leqq t \leqq 1,
\end{aligned}
$$

for all $(x, t) \in\left(X K_{2}^{*}\right) \times[0,1]$. Note that $F^{*}(x, 0)=x$ and $F^{*}(x, 1)=$ $g \circ F^{\prime}(f(x), 1) \in g\left(K_{2}^{\prime}\right)$, for all $x \in X \backslash K_{2}^{*}$. If $K_{2}$ is a compact set in $X$ containing $g\left(K_{2}^{\prime}\right) \cup K_{2}^{*}$ and $F=F^{*} \mid\left(X \backslash K_{2}\right) \times[0,1]$, then we have $F(x, 1)=$ $g \circ F^{\prime}(f(x), 1) \in g\left(K_{2}^{\prime}\right) \subset K_{2}$, for all $x \in X \backslash K_{2}$. Thus we have constructed $K_{2}$ and $F$ to fulfill our requirements.

3. The example. We will first need to describe Whitehead's example of an open contractible 3-manifold which is topologically different from $E^{3}$ [7]. We use the description of Bing [2]. Consider a solid torus $T_{1}$ embedded in a solid torus $T_{2}$ as shown in the figure below (where $T_{2}$ is a subset of $E^{3}$ ). Since $T_{1}$ is tame in $E^{3}$ there exists a homeomorphism $h$ of $E^{3}$ onto itself such that $h\left(T_{1}\right)=T_{2}$. Let $M=T_{1} \cup h\left(T_{1}\right) \cup h^{2}\left(T_{1}\right) \cup \cdots$, which 
is Whitehead's open contractible 3-manifold. Lei $T_{n+1}$ be the torus $h^{n}\left(T_{1}\right)$, for all $n \geqq 2$.

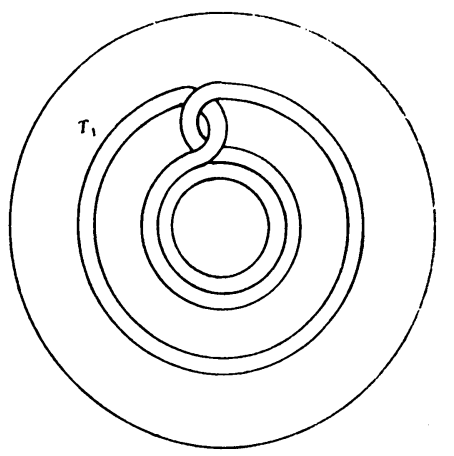

In [7] Whitehead proved that $M$ is not PL homeomorphic to $E^{3}$. In [6] Newman and Whitehead examined the fundamental groups of various subsets of $M$ and used this information to prove that $M$ is not homeomorphic to $E^{3}$. As a consequence of the work in [6] we have the following:

1. $\pi_{1}\left(M \backslash T_{1}\right)$ is not finitely generated $(f . g$.$) .$

2. $\left.\pi_{1}\left(T_{n}\right\rangle T_{1}\right)$ is f.g., for all $n>1$.

Since $M$ is a manifold it is obvious that $M \times Q$ is a $Q$-manifold, therefore a contractible $Q$-manifold (since $M$ is contractible). We now proceed to prove that $M \times Q$ is not of type $Q$. We do this by showing that the assumption that $M \times Q$ is of type $Q$ leads to a contradiction. The assumption that $M \times Q$ is of type $Q$ implies that $M \times Q$ is proper homotopically dominated by $Q \backslash A$, for some closed $A \subset W$. Since $Q$ is compact and contractible, it easily follows that $M$ and $M \times Q$ have the same proper homotopy type. Thus $M$ is proper homotopically dominated by $Q \backslash A$. Using Lemma 2.1 there exists a compact set $C \subset M$ containing $T_{1}$ and a map $F:(M \backslash C) \times[0,1] \rightarrow M \backslash T_{1}$ such that $F(x, 0)=x$ and $F(x, 1) \in C \backslash T_{1}$, for all $x \in M \backslash C$.

Now choose $n>1$ large enough so that $C \subset T_{n}$. It is obvious that $\partial T_{n}=$ $\operatorname{Bd}_{M I}\left(T_{n}\right)$ is collared in $T_{n} \mid C$, i.e. there exists an embedding $f: \partial T_{n} \times$ $[0,1] \rightarrow T_{n}: C$ such that $f\left(\partial T_{n} \times[0,1)\right)$ is open in $T_{n}$ and $f(x, 0)=x$, for all $x \in \partial T_{n}$. We now define a map $G:\left(M \backslash T_{1}\right) \times[0,1] \rightarrow M \backslash T_{1}$. To define $G$ on $\left(M \backslash f\left(\partial T_{n} \times(0,1)\right)\right) \times[0,1]$ we put

$$
\begin{aligned}
G(x, t) & =x & & \text { for } x \in T_{n} f\left(\partial T_{n} \times[0,1) i, t \in[0,1],\right. \\
& =F(x, t) & & \text { for } x \in M
\end{aligned}
$$

To define $G$ on $f\left(\partial T_{n} \times[0,1]\right) \times[0,1]$ choose any $x \in \partial T_{n}$ and put $G(f(x, s), t)=F(f(x, s),(1-s) t)$, for all $s, t \in[0,1]$. It is clear that $G$ satisfies $G(x, 0)=x$, for all $x \in M \backslash T_{1}$. Since $F\left(f\left(\partial T_{n} \times[0,1]\right) \times[0,1]\right)$ is 
a compact subsel of $M: T_{1}$ there exists an integer $m \geqq n$ such that $F\left(f\left(\partial T_{r_{t}} \times[0,1]\right) \times[0,1]\right) \cdots T_{m} T_{1}$. It then follows that $G(x, 1) \in T_{m} \backslash T_{1}$, for all $x \in M: T_{1}$.

Choose any $x_{0}: C i T_{1}$ and let $\left.\sigma: I \rightarrow M\right) T_{1}$ be a loop at $x_{0}$, i.e. $\sigma: I \rightarrow$ $M \backslash T_{1}$ is a map and $\sigma(0)=\sigma(1)=x_{0}$. For each $t \in[0,1]$ define a loop $\sigma_{t}: I \rightarrow M \backslash T_{1}$ by $\sigma_{t}(s)=\left(j(\sigma(s), t)\right.$, for all $s \in[0,1]$. Then each $\sigma_{t}$ is a loop at $x_{0}, \sigma_{0}=\sigma$, and $\sigma_{1}$ is a loop in $T_{m} \backslash T_{1}$. This proves that each loop in $M \backslash T_{1}$ at $x_{0}$ can be continuously deformed to a loop in $T_{m} \backslash T_{1}$ at $x_{0}$ (with the point $x_{0}$ being kept fixed at each level of the deformation). Restating this it means that if $i: T_{m} T_{1} \rightarrow M T_{1}$ is the inclusion map, then

$$
i_{*}: \pi_{1}\left(T_{m}: T_{1}, x_{0}\right) \rightarrow \pi_{1}\left(M: T_{1}, x_{0}\right)
$$

is a surjection. But this contradicts the facts that $\pi_{1}\left(T_{m} T_{1}\right)$ is f.g. and $\pi_{1}\left(M \backslash T_{1}\right)$ is not f.g.

\section{RFFERENCES}

1. R. D. Andersoli, T. A. (hapman and R. M. Schori, Problems in the topology of infinite-dimensional pur and manifolds, ZW-report, Department of Pure Mathematics, Mathematical enter, Amsterdam, 1971.

2. R. H. Bing, ve:ssary and sufficient conditions that a 3-manifold be $S^{3}$, Ann. of Math. (2) 68 (1958), 17-37. MR 20 \#1973

3. T. A. Chapman, On the structure of Hilbert rube manifolds, (ompositio Math. (to appear).

4. ...... Hilhert ' wre manifold;, Bull. Amer. Math. Soc. 76 (1970). 1326-1330.

5. -......-, On some applic ations of infinite-dimensional manifolds to the theory of shape, Fund. Math. (to appear)

6. M. H. A. Newmian and J. H. C. Whitehead, On the group of a rertain linkage, Quart. J. Math. 8 (19:7), 1421

7. J. H. C. Whitehearl, A ertain open manifold "hose group is unity, Quart. J. Math. $6(1935), 268 \cdot 27 \%$

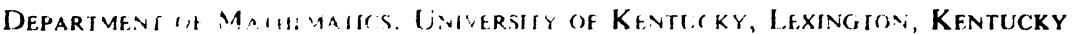
40506 